\title{
Relationship between oxidative stress and nuclear factor-erythroid-2-related factor 2 signaling in diabetic cardiomyopathy (Review)
}

\author{
XIA WU ${ }^{1,2}$, LEITAO HUANG ${ }^{3}$ and JICHUN LIU ${ }^{1,2}$ \\ ${ }^{1}$ Department of Pharmacy, Nanchang University; ${ }^{2}$ Department of Thoracic and Cardiovascular Surgery, \\ The First Affiliated Hospital of Nanchang University, Nanchang, Jiangxi 330006; ${ }^{3}$ Department of Orthopedics, \\ The Fourth Affiliated Hospital of Nanjing Medical University, Nanjing, Jiangsu 210031, P.R. China
}

Received January 30, 2020; Accepted August 26, 2020

DOI: $10.3892 /$ etm.2021.10110

\begin{abstract}
Diabetic cardiomyopathy (DCM) is the leading cause of death worldwide, and oxidative stress was discovered to serve an important role in the pathophysiology of the condition. An imbalance between free radicals and antioxidant defenses is known to be associated with cellular dysfunction, leading to the development of various types of cardiac disease. Nuclear factor-erythroid-2-related factor 2 (NRF2) is a transcription factor that controls the basal and inducible expression levels of various antioxidant genes and other cytoprotective phase II detoxifying enzymes, which are ubiquitously expressed in the cardiac system. Kelch-like ECH-associated protein 1 (Keap1) serves as the main intracellular regulator of NRF2. Emerging evidence has revealed that NRF2 is a critical regulator of cardiac homeostasis via the suppression of oxidative stress. The activation of NRF2 was discovered to enhance specific endogenous antioxidant defense factors, one of which is antioxidant response element (ARE), which was subsequently illustrated to detoxify and counteract oxidative stress-associated DCM. The NRF2 signaling pathway is closely associated with the development of various types of cardiac disease, including ischemic heart disease, heart failure, myocardial infarction, atrial fibrillation and myocarditis. Therefore, it is hypothesized that drugs targeting this pathway may be developed to inhibit the activation of NRF2 signaling, thereby preventing the occurrence of DCM and effectively treating the disease.
\end{abstract}

Correspondence to: Professor Jichun Liu, Department of Thoracic and Cardiovascular Surgery, The First Affiliated Hospital of Nanchang University, 17 Yong Wai Zheng Street, Nanchang, Jiangxi 330006, P.R. China

E-mail: 504716243@qq.com

Key words: cardiac diseases, oxidative stress, nuclear factor-erythroid-2-related factor 2, antioxidant response element, diabetic cardiomyopathy, Kelch-like ECH-associated protein 1

\section{Contents}

1. Introduction

2. The NRF2/Kelch-like ECH-associated protein 1 (Keap1)/ARE signaling pathway

3. NRF2 signaling and its association with DCM complications

4. Natural substance therapies for DCM

5. miRNAs as a novel drug therapy for DCM

6. Conclusions

\section{Introduction}

Diabetic cardiomyopathy (DCM) is widespread, as is the necrosis of the myocardium caused by diabetes mellitus (DM), and leads to cardiac microangiopathy and myocardial metabolic disorders (1). Early diastolic dysfunction is usually characterized by decreased myocardial compliance and blocked diastolic filling (2). Late diastolic dysfunction is mainly manifested as congestive heart failure (HF) (3). DCM is one of the main complications of DM, differing from hypertension and other types of cardiovascular disease, which demonstrates one of the highest incidence rates of all cardiovascular diseases with an incidence rate of $16.9 \%$ in China (4). In fact, DCM accounts for $>80 \%$ of the deaths of diabetic patients $(5,6)$.

A large number of studies have reported that the pathogenesis of DCM primarily involves mitochondrial dysfunction, impaired intracellular calcium regulation, the accumulation of advanced glycation end products in the heart, abnormal cell metabolism and endoplasmic reticulum stress $(2,7)$. Mitochondrial dysfunction has been discovered to lead to the excessive production of reactive oxygen species (ROS), which in turn can promote the opening of mitochondrial permeability transition pores (mPTP), reduce the mitochondrial membrane potential and impede the respiratory transmission chain (8). Impaired intracellular calcium regulation has been identified to lead to impaired cardiac contractility, while the accumulation of advanced glycation end products in the heart was discovered to lead to a buildup of extracellular matrix, 
which in turn leads to diastolic dysfunction and eventually to functional failure (9). In addition, abnormal cell metabolism was shown to lead to the accumulation of toxic lipids in the heart, and endoplasmic reticulum stress is known to mediate cell apoptosis. It is also well known that metabolic disorders cause subcellular inflammation of the heart and inflammation is an important pathogenic feature of DM (Fig. 1) (10).

These pathologies are mainly caused by hyperglycemia, hyperlipidemia and inflammation, which can stimulate the production of ROS or reactive nitrogen species (11). ROS are small, highly reactive molecules, also known as free radicals, which serve important roles in pathology and physiology. The role of the heart is to provide sufficient blood flow to organs and tissues to supply them with oxygen and various nutrients. The heart has high energy requirements and is prone to oxidative damage caused by physiological processes (12). The heart needs more oxygen than other organs and consumes more energy (6). It has been reported that oxidative stress served an important role in various types of cardiac disorder (13). Oxidative stress is a negative condition caused by the production of free radicals, and manifests as an imbalance of oxidation and antioxidant effects in the body (14). The higher the levels of ROS in the heart, the higher the amount of oxidative stress produced (15). ROS is considered to be responsible for systolic and endothelial dysfunction, cardiac cell apoptosis and necrosis (16). Therefore, the reduction of oxidative stress is an attractive target for the treatment of cardiac diseases.

Nuclear factor-erythroid-2-related factor 2 (NRF2) is expressed in a wide range of tissues and organs, including the heart, brain, liver, kidney and skin (17). A large number of reports have indicated that NRF2 signaling served important roles in processes, such as embryonic development, oxidative stress and ischemia/reperfusion injury (IRI) (14). NRF2 was also reported to regulate the clearance of free radicals and lipid homeostasis (18). Therefore, NRF2/antioxidant response element (ARE) signaling has become an attractive target for the treatment of DCM. The NRF2 signaling pathway is reportedly involved in DCM via the transcriptional regulation of other signaling pathways, including PI3K/AKT and JAK/STAT (19). The present review aimed to provide a comprehensive discussion of the current understanding of the regulation of NRF2-mediated signaling in the development of DCM.

\section{The NRF2/Kelch-like ECH-associated protein 1 (Keap1)/ARE signaling pathway}

Under the appropriate stimulation by physical and chemical factors, including inflammation, trauma and fever, NRF2 dissociates from Keap1, which permits the levels of NRF2 degradation to decrease and the simultaneous synthesis of NRF2 to increase, which subsequently facilitates NRF2 entering the nucleus, where it specifically recognizes and binds to the core sequence of AREs (20). As a result of this series of actions, multiple downstream antioxidants are activated, where the expression levels of inflammatory proteins and detoxification enzymes are upregulated (21). The transcription of these genes, including superoxide dismutase (SOD), GSH, GPX and heme oxygenase (HO-1), has been identified to suppress ROS production and reduce oxidative stress (Fig. 2) (22).
NRF2, which contains 589 amino acids, is a basic leucine zipper (bZIP) transcription factor belonging to the Cap 'n' Collar family that contains seven functional domains, Neh1-Neh7 (23). These structures serve an important role in regulating the stability of NRF2 (24). The bZIP structure of the Nehl region can bind to the small Maf protein to form a heterodimer, which subsequently binds to the DNA molecule through the ARE (25). Neh2 is the region in which NRF2 binds to Keap1; the region involves the 69 to 84th amino acids of NRF2 (a short peptide of 16 amino acids), which contains an ETGE motif and a DLG motif binding site that binds to Keap1 (26). The Neh3 domain is located at the carboxy terminus and is highly conserved (27). The agonist chromatin helicase DNA binding protein 6 binds to the Neh3 domain and upregulates NRF2 target gene expression (Fig. 3) (28). The Neh4 and Neh5 domains, which are related to the 596 and 599 amino acids of NRF2, are involved in the transcriptional activation of the NRF2 target gene after binding to the coactivator cAMP-response element binding protein (CREB) (29). The Neh6 region contains a large number of serine residues, which form redox-insensitive regions. Under oxidative stress conditions, the degradation of Nrf2 is associated with Neh6, such that Neh6 mediate B-TrCP-dependent ubiquitination of the NRF2 transcription factor so that NRF2 can be degraded (30). Finally, the Neh7 domain can inhibit NRF2 by binding to the retinoic $\mathrm{X}$ receptor $\alpha$ (31).

Keap1 is an important factor regulating the NRF2 response and a central regulator of cellular oxidative stress. Keap1 contains five domains: i) An N-terminal region domain; ii) a bric á brac (BTB) dimerization domain; iii) an intervening region (IVR) domain; iv) a theme containing the six Kelch motifs; and v) a C-terminal region domain (32). BTB, which is an evolutionarily conserved motif involved in protein-protein interactions found in actin-binding proteins and zinc finger transcription factors, usually forms a dimer with other BTB regions; this domain is required for Keap1 to dissociate from NRF2 and prevent phase II gene transcription (33). The IVR domain is rich in cysteine and contains the most active cysteine residue of Keapl; it is a functional regulatory region for the entire protein, which is involved in the reactions of electrophilic compounds and oxidants, and also participates in the formation of ubiquitination linkages to stabilize NRF2 (31). The DGR domain contains six bis-glycine repetitive sequences or six Kelch motifs; the repetitive Kelch motifs form six classical p-helices with multiple potential protein binding sites for Keapl to bind to the Neh2 region, and this is where Keapl also binds to cytoplasmic actin (34).

The ARE is the core protector of cytoprotective proteins, representing an important antioxidant component of the human body (35). Under reactive chemical pressure, ARE cis-acting elements function primarily at the transcriptional level to regulate the expression levels of numerous cytoprotective enzymes, including SOD and HO-1 (36). The ARE has important structural and biological characteristics and demonstrates a unique ability to respond to oxidative stress (37). It reacts not only to $\mathrm{H}_{2} \mathrm{O}_{2}$, but also to specific chemical compounds, and has the ability to conduct redox cycles or produce reactive or electrophilic intermediates (38). A number of compounds have a tendency to react with the sulfhydryl groups, such as diethyl maleate, 


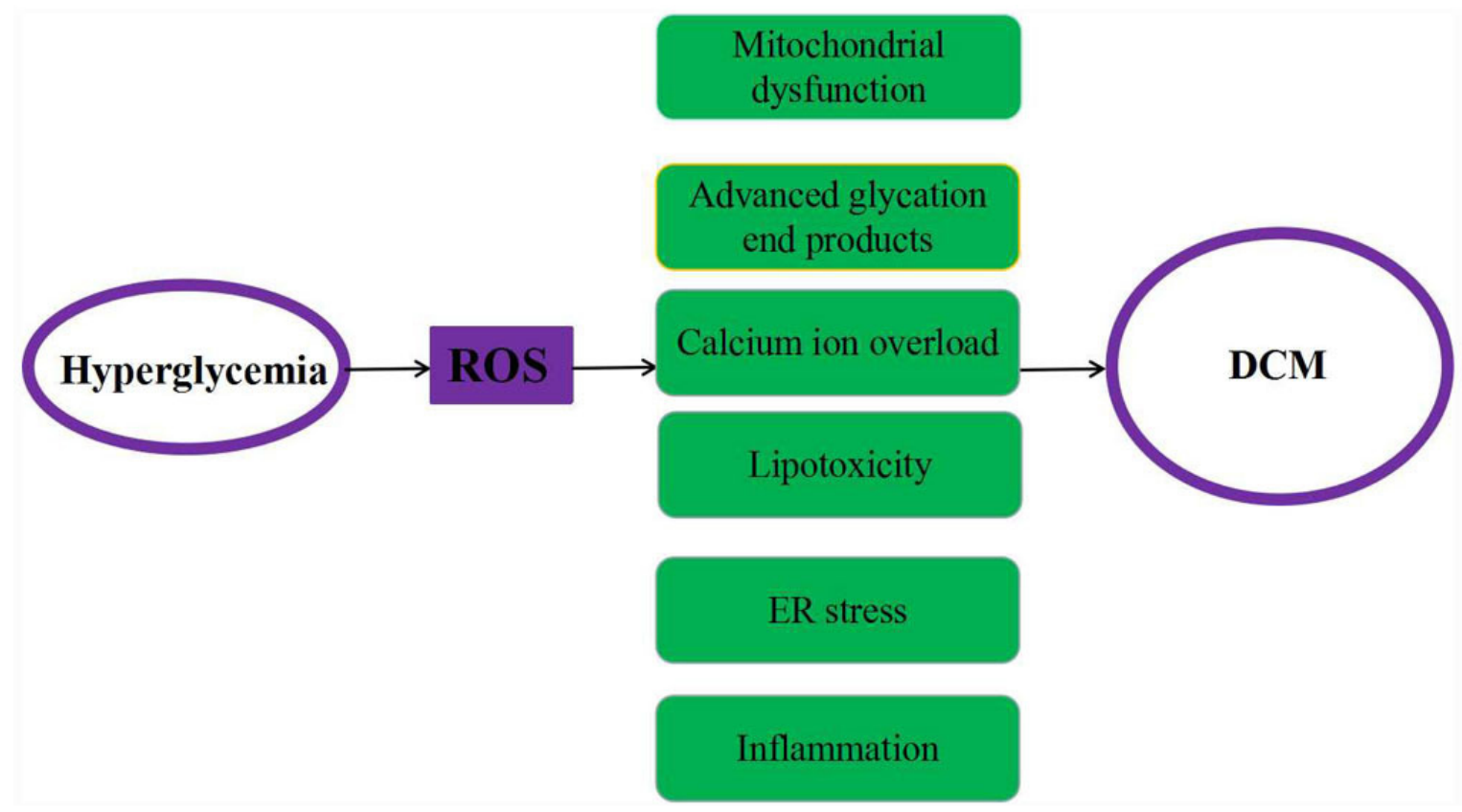

Figure 1. Pathogenesis of DCM. The consequence of long-term hyperglycemia is that the heart tissue will produce a large amount of ROS, which will induce a series of physiological changes in cardiomyocytes, including mitochondrial dysfunction, advanced glycation end products, calcium overload, lipotoxicity, ER stress and inflammation. This will eventually develop into DCM. DCM, diabetic cardiomyopathy; ROS, reactive oxygen species; ER, endoplasmic reticulum.

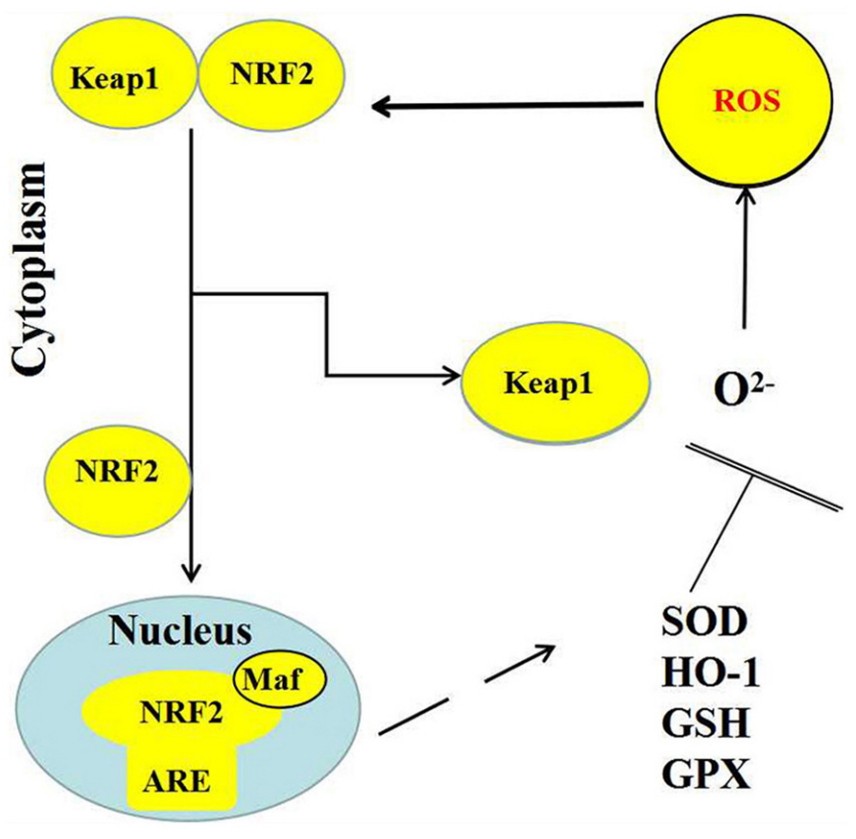

Figure 2. NRF2 exerts antioxidant activity in the presence of ROS. NRF2, nuclear factor-erythroid-2-related factor 2; Keap1, Kelch-like ECH-associated protein 1; ROS, reactive oxygen species; ARE, antioxidant response element; SOD, superoxide dismutase; GSH, glutathione; CAT, catalase; GPX, glutathione peroxidase.

dithiothiones and isothiocyanates, which can potently induce ARE activity $(39,40)$.

NRF2 is a key regulator of antioxidant reactions and has been demonstrated to regulate both oxidative stress and inflammation $(41,42)$. There are two types of antioxidant systems in the body: One is the enzyme antioxidant system, which includes superoxide, SOD, catalase and glutathione S-transferase; the other system is a non-enzymatic antioxidant system, involving, for example, glutathione (43). NRF2/Keap1 signaling serves an important role in the maintenance of intracellular redox homeostasis, in addition to serving a vital role in a variety of cell types and organs in different types of oxidative damage-related cardiac disease, including myocardial ischemic disease, HF and cardiac hypertrophy (44). Numerous NRF2 activators are plant-derived phytochemicals and natural products, such as curcumin (CUR), sulforaphane (SFN) and resveratrol (RES) compounds (45), although some synthesized NRF2 activators have been identified, including hydrogen sulfate and 4-hydroxynonenal lipoic acid (46). These chemoprotective compounds protect cells from oxidative stress by mediating the NRF2 defense response, and activating phase II detoxification enzymes, transporters and antioxidants (47)

NRF2/ARE signaling has been associated with various major pathophysiological conditions, including hypoxia, ischemia, fibrosis and apoptosis (48). It is also associated with numerous signaling pathways (49). There is increasing evidence to suggest that NRF2 and PI3K/AKT are important in oxidative stress injury, where NRF2 is involved in several signaling pathways, including the $\mathrm{NF}-\kappa \mathrm{B}$ and other cytokine signaling pathways (50). Therefore, NRF2/ARE signaling is known to serve an important role in several pathological conditions.

\section{NRF2 signaling and its association with DCM complications}

Ischemic heart disease. Ischemic heart disease is a serious condition, which among all patients with heart disease in China the death rate can reach $60 \%$ (51). In myocardial IR, the blood supply to the heart is blocked, following which perfusion and accompanying reoxygenation resumes (52). IR occurs 


\begin{tabular}{|c|c|c|c|c|c|c|c|c|c|}
\hline \multirow[b]{2}{*}{ NRF2 } & \multirow[b]{2}{*}{ H2N } & \multirow{2}{*}{$\begin{array}{l}\begin{array}{l}\text { Keap1 } \\
\text { binding }\end{array} \\
\text { Neh2 }\end{array}$} & \multicolumn{2}{|c|}{$\begin{array}{l}\text { Transactivation } \\
\text { domain }\end{array}$} & \multirow{2}{*}{$\begin{array}{l}\begin{array}{l}\text { RxRa } \\
\text { binding }\end{array} \\
\text { Neh7 }\end{array}$} & \multirow{2}{*}{$\begin{array}{l}\beta \text {-TrCP } \\
\text { binding } \\
\text { Neh6 }\end{array}$} & \multicolumn{2}{|c|}{$\begin{array}{l}\text { DNA binding } \\
\text { protein } 6\end{array}$} & \multirow[b]{2}{*}{$\mathrm{COOH}$} \\
\hline & & & Neh4 & Neh5 & & & Neh1 & Neh3 & \\
\hline
\end{tabular}

Cul3 binding Kelch domain DGR domain

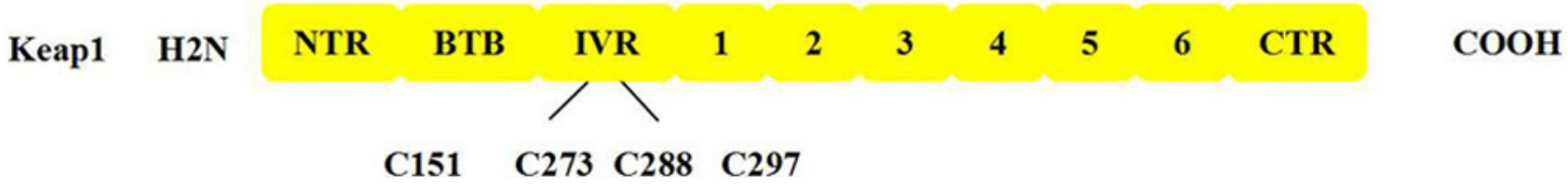

Figure 3. Domain structure of NRF2 and Keap1. NRF2, nuclear factor-erythroid-2-related factor 2; Keap1, Kelch-like ECH-associated protein 1; $\beta$-TrCP, $\beta$-transducin repeat containing; NTR, N-terminal region; BTB, bric á brac; IVR, intervening region; C-terminal region; RxRa, retinoid X receptor $\alpha$; Cul3, cullin 3 .

in acute coronary syndrome, cardiopulmonary resuscitation, organ transplantation thrombolysis, coronary bypass and other conditions (53). Calcium and ROS were discovered to be important molecular species involved in myocardial IRI during ischemia initiation and reperfusion. Calcium has an important electrophysiological elements, which enhances myocardial vitality and participates in heartbeat, whilst ROS plays an important regulatory role during myocardial ischemia and reperfusion (39). The mPTP opening is an important mechanism of myocardial necrosis induced by IRI (22). However, due to the complicated underlying mechanisms, there are currently no effective methods for preventing myocardial IRI.

The most critical feature of myocardial IRI formation is the production of ROS (54). During perfusion in the ischemic phase, residual oxygen can still reach the tissue and produce ROS in the myocardium (55). A small amount of ROS is formed in the early stages of myocardial ischemia, but levels were found to increase sharply after 20-25 min (56). Myocardial reperfusion is extremely sensitive to large increases in ROS, which not only damage the mitochondrial respiratory chain, but also oxidize the mitochondrial inner membrane cardiolipin and inhibit respiratory chain activity (57). When ROS levels are increased, they can also induce lipid peroxidation and cardiomyocyte oxidative damage, eventually leading to apoptosis (58).

NRF2 regulates a series of antioxidant enzymes and cell oxidative stress, and it has been reported that the overexpression of NRF2 protected against IRI (59). The myocardial infarction (MI) area was demonstrated to increase, while the degree of cardioprotection decreased in NRF2 knockout mice following IR (60). Notably, following the activation of the NRF2 signaling pathway, a large number of compounds can protect the heart from IR damage (61). Similarly, reduced NRF2 activity in H9C2 cardiomyoblasts led to a decrease in cell survival under hypoxic conditions, irrelevant of whether reoxygenation occurred (62).

It has been suggested that NRF2 activity may be linked to mitochondrial function (63). NRF2 signaling controlled mitochondrial ROS production by regulating mitochondrial function, which indicated that the NRF2 signaling pathway may serve a vital role in cellular redox homeostasis (64). In clinical terms, this may lead to improved ischemic tolerance and make organs more resistant to ischemia (65). Following ischemic injury, organ transplantation and IR are beneficial for restoring blood flow (66). Although it is also worthy to note that several drugs can also be used to prevent IRI (67), as it is important to remove ROS from cardiomyocytes, the manipulation of the NRF2/Keap1 signaling pathway may represent a novel strategy for the treatment of IRI (68).

$H F$. HF is a pathological process in which the cardiac pump function is reduced due to myocardial contraction and/or diastolic dysfunction, resulting in a decrease in cardiac output and an inability of the heart to meet the metabolic needs of the body tissues (69). HF is considered the end point of numerous types of cardiovascular disease. Persistent, abnormal neurohormonal and mechanical stresses can lead to HF (70); such pathological stresses include, hypertension, cardiac fibrosis, cardiac hypertrophy and cardiomyocyte apoptosis (71). These stresses can alter the microvascular structure and ventricular dilation, ultimately leading to cardiac dysfunction and HF $(12,38)$. However, it is common that the heart remains within the normal range of ejection fraction (72).

It was previously reported that oxidative stress accelerated the progression of HF (73). In fact, there is some experimental evidence to suggest that ROS-induced changes in the structure and function of the heart can lead to HF, which highlights that oxidative stress and myocardial function are closely related. 
NRF2 is known to be associated with the inducible expression of various antioxidant genes and other cytoprotective phase II detoxifying enzymes, and it was previously reported that NRF2 participated in the mechanism of $\mathrm{HF}$ (74-76). In addition, several clinical studies have identified that microRNA (miRNA/miR)-27a expression levels were upregulated in human $\operatorname{HF}(63,67,77)$. This is interesting as miR-27a was one of the microRNAs predicted to be a target of NRF2; however, its mechanism of action remains unknown $(69,78)$. Therefore, it is important to further investigate the specific role of NRF2 during HF.

Cardiac remodeling. Cardiac remodeling is an adaptive process of cardiomyocytes to repeated stress in order to maintain homeostasis (79). Cardiac remodeling frequently impairs function at the level of molecules, cells, tissues and organs (80). Numerous animal and clinical studies have revealed that cardiac hypertrophy, myocardial apoptosis and interstitial collagen deposition eventually led to systolic dysfunction (81-83).

During cardiac remodeling, the loss of cardiomyocytes has been widely ascribed to necrosis, apoptosis or autophagy (80); however, fibrosis can also occur through fibroblast proliferation and extracellular matrix reorganization (84). Mitochondrial dysfunction and metabolic abnormalities were also discovered to contribute to the process of cardiac remodeling $(85,86)$. In fatigued cardiomyocytes, calcium uptake is impaired, where calcium efflux becomes dysregulated and involves components such as sarco/endoplasmic reticulum calcium-ATPase-2a and the lysine receptor (87). Morphological changes also occur in the heart, with the shape morphing from elliptical to spherical (82). Cardiac remodeling also damages the systolic function of the heart (88).

Cardiac remodeling usually leads to left ventricular mass hypertrophy and a reduction of ejection fraction (89). It has been previously reported that ROS activated NRF2 signaling to facilitate different cardiac remodeling processes (90). Notably, the activation of the NRF2 pathway using allicin treatment was observed to prevent the development of cardiac remodeling to cardiac dysfunction (91). Oxidative stress was also discovered to be closely related to cardiac remodeling (92). In addition, antioxidants have been found to prevent cardiac remodeling and several studies have revealed that oxidative stress served a regulatory role in myocardial fibrosis $(86,88,93)$. Therefore, NRF2 may serve as a good potential target for cardiac remodeling.

MI. MI is a type of myocardial necrosis caused by the acute and persistent ischemia and hypoxia of the coronary arteries, which occurs due to an imbalance between the oxygenated blood supply and demand (92). Every year, $10 \%$ of patients with symptoms of acute coronary syndrome are diagnosed with acute MI worldwide (94). Necrosis was considered to be the main cause of cardiomyocyte death; however, cell apoptosis also serves an important role in the process of cell death (95). Although rapid reperfusion therapy was discovered to reduce infarct size and improve left ventricular function, reperfusion itself resulted in cell necrosis (96). IRI was also revealed to serve an important role in oxidative stress and myocardial infarction (97). During oxidative stress, large amounts of ROS are produced, which was identified to induce lipid peroxidation and convert oxidizing proteins to an inactive state, resulting in DNA damage and the exacerbation of IRI (11). Therefore, excess ROS should be eliminated as soon as possible. NRF2 is a well-known antioxidant and has been discovered to be closely related to MI (51). NRF2/Keap1 pathway is an important antioxidant defense mechanism, which is closely related to DCM heart remodeling mediated by oxidative stress (98). It has been reported that after myocardial infarction (MI), the NRF2 protein in the heart is downregulated, which leads to a decrease in the antioxidant enzymes targeted by Nrf2 (91). At this stage, the human body will protect the heart tissue from further damage, so it will trigger a series of reactions to downregulate NRF2 (96). As the heart tissue continues to undergo irreversible damage, DCM will increase the transcription and activation of NRF2 (14). Therefore, if a gene target that blocks Nrf2 is found, it may provide a new method for the treatment of DCM $(96,98)$.

Cardiac hypertrophy. Cardiac hypertrophy is a relatively slow, but effective, compensatory function, which occurs mainly under long-term stress overload (99). Cardiac hypertrophy includes physiological hypertrophy and pathological hypertrophy (100). Physiological hypertrophy occurs mainly in healthy individuals, pregnant women and individuals pursuing long-term high intensity training and it is a reversible condition; however, pathological hypertrophy is a compensatory response (101). Different stimuli can provoke different types of cardiac hypertrophy, including persistent stress overload (hypertension), excessive volume overload (arteriovenous shunt) and genetic mutations (cardiomyopathic hypertrophy) (102).

Cardiomyocytes can enhance myocardial contractility to a certain extent under the stimulus of various outside factors, including myocardial ischemia and septic shock (103). When a stimulus persists, the compensatory mechanisms of the myocardial cells become decompensated, and eventually HF develops (80). Cardiac hypertrophy has been strongly linked to $\mathrm{HF}$ and cardiac death, indicating that preventing cardiac hypertrophy may be of utmost importance (53). Previous studies revealed that oxidative stress activated cardiac hypertrophy and other pathways including PI3K/AKT, NRF2/ARE signaling (104). Notably, upon the knockdown of NRF2 expression, angiotensin II-induced cardiac hypertrophy was exaggerated and the degree of oxidative stress in the heart was exacerbated (105). Other previous studies have indicated that endogenous antioxidants, such as $\mathrm{HO}-1$, protected against cardiac hypertrophy $(22,68)$. For example, in hypertension model rats, an increase in HO-1 effectively reduced left ventricular hypertrophy (106-108).

Myocarditis. Myocarditis can be caused by viral, bacterial or parasitic infections, or by noninfectious factors, including trauma and drugs (41). Therefore, myocarditis is a common infectious or non-infectious myocardial immunopathological process (109). Acute viral myocardial inflammatory injury without clinical symptoms and chronic myocarditis were discovered to lead to immune-mediated myocardial damage and dysfunction (110). Importantly, myocarditis is a precursor lesion of DCM and may develop into DCM. It has been 
reported that ROS and oxidative stress were involved in the pathogenesis of myocarditis (78). ROS and oxidative stress are associated with innate and adaptive immunity and tissue repair (73).

Persistent myocardial inflammation causes myocardial remodeling, which eventually develops into DCM. This leads to the release of cytokines, causing an inflammatory response. Histamine was discovered to increase the susceptibility of mice to autoimmune myocarditis (111). In addition, the activation of cytokines, such as TGFs, activated the intracellular signaling protein SMAD cascade, increased profibrotic factors, pathological fibrosis and myocardial remodeling, and decreased cardiac function, all of which lead to progressive HF (112). ROS was also identified to directly alter cardiac contractility; it has also been associated with cardiac depression in acute inflammation other than chronic myocarditis (113).

NRF2 is an important regulator of antioxidant reactions, which regulate oxidative stress (12). Several natural compounds, such as CUR, have antioxidant properties (53). CUR was discovered to regulate specific transcription factors, such as NRF2, which subsequently regulated free radical scavenging and the expression of lipid homeostasis (102). The induction of the activity of antioxidative enzymes has been attempted using gene therapies and nanotechnologies (39). However, there is a lack of clinical evidence for this effect $(71,114)$. The clinical efficacy has not been established in human myocarditis, therefore, further research remains to be undertaken. Although additional studies are required, NRF2 may serve a vital role in myocarditis.

\section{Natural substance therapies for DCM}

Oxidative stress, and hence antioxidants, have been illustrated to serve an important role in the pathogenesis of DCM (82). NRF2 and its downstream signaling pathways have been reported to have a key role in preventing DCM and other cardiovascular events induced by high glucose (69). In addition, it was demonstrated that natural or synthetic NRF2 activators had a therapeutic role in DCM model animals (54). Therefore, NRF2 is considered to be a target for the treatment of DCM.

CUR. CUR is a naturally occurring polyphenol isolated from the turmeric plant (115); it has been discovered to contain antioxidants, anti-inflammatory agents and anti-cancer agents, in addition to exerting anti-diabetic activity (65). In streptozocin (STZ)-induced diabetic model mice, CUR intervention was identified to reduce blood glucose levels (116). Using malondialdehyde and NAD(P)H quinone dehydrogenase 1 (NQO1) as indicators, CUR was also reported to inhibit myocardial oxidative stress by upregulating NRF2 in type 1 DM (T1DM) model rats (45). The upregulation of the endogenous antioxidant gene NRF2 also inhibited hyperglycemia-induced inflammation, macrophage infiltration, diabetic-induced cardiac dysfunction, endoplasmic reticulum stress, oxidative stress injury and apoptosis (117).

$S F N$. As an isothiocyanate, $\mathrm{SFN}$ is widely found in cruciferous vegetables, especially broccoli (118). SFN was discovered to be an activator of the transcription factor NRF2, and it was observed to reduce the oxidative damage caused by DCM and improved cardiac function in T1DM model mice (119). SFN was also identified to effectively prevent cardiac enlargement and cardiac dysfunction in type $2 \mathrm{DM}$ model mice by upregulating the expression levels of NRF2 and the subsequent expression levels of the downstream genes, HO-1 and NQO1, and significantly reducing the myocardial inflammatory response, fibrosis and oxidative damage (120).

RES. RES is a polyphenolic substance widely present in plants, including grape skins, nuts and knotweed (121). It was shown to have antioxidative stress and antihypertensive effects in T1DM model rats (122). RES was found to attenuate cardiac dysfunction, cardiac hypertrophy and myocardial fibrosis (123). By detecting various indicators of oxidative stress in the heart, it was also illustrated that RES significantly reduced the degree of oxidative stress damage caused by DM (124). RES also reduced oxidative stress and inhibited the cardiac hypertrophy and vascular fibrosis caused by connective tissue growth factor by upregulating NRF2 expression levels and the transcriptional activity of its downstream target genes (125).

$D M F$. DMF is an oral drug used for the treatment of adult multiple sclerosis and in Europe for the treatment of psoriasis (126). In both human and animal experiments, it was demonstrated that DMF effectively activated NRF2 and promoted its downstream antioxidative stress activity (127). In mice with T1DM, the measurements of the antioxidant enzymes, SOD, HO-1 and other indicators, suggested that DMF significantly inhibited the levels of oxidative stress in the DCM myocardium by reducing the source of free radicals and increasing the expression of enzymes and proteins involved in oxidative stress (128). Another previous study also revealed that the protective effect of DMF on DCM was related to the upregulation of NRF2 and the expression of downstream antioxidant genes in the cardiomyocytes of T1DM model mice (129).

Rutin. Rutin is a medicinally active ingredient of a flavonoid plant that exerts strong pharmacological activity and low toxicity (130). Rutin reportedly promoted vasodilation, the antagonization of platelet activating factor, anti-inflammatory and anti-oxidative processes, and the protection of the pancreas (131). It was reported that rutin may also reverse diabetic myocardial injury (78). In STZ-induced diabetic model mice, the levels of serum myocardial enzymes and other indicated were recorded, and hematoxylin and eosin and Masson's trichrome staining were performed, and rutin was illustrated to reduce the serum myocardial enzyme content, improve the pathological morphology of myocardial cells, reduce the degree of fibrosis and alleviate myocardial injury in mice with DCM (132).

\section{5. miRNAs as a novel drug therapy for DCM}

miRNA is a type of endogenous, non-coding single-stranded RNA, which participates in numerous physiological processes, including angiogenesis, metabolism, cell growth, survival and death, proliferation and differentiation (133). miRNA can negatively regulate gene expression by 
promoting the degradation, and inhibiting the translation, of target RNA (134). Notably, the dysregulation of miRNA biological mechanisms has been shown to promote a variety of diseases, including DCM (135). For example, miR-1 was found to be abundant in the diabetic myocardium, where it promoted ROS production and the decline in mitochondrial membrane potential, whilst upregulating Bax expression levels and inhibiting $\mathrm{Bcl}-2$ expression levels, thereby promoting cardiomyocyte apoptosis (136). Similarly, Dludla et al (137) suggested that inhibiting the expression levels of miR-1 had a protective effect on the myocardium in patients with diabetes.

As one of the important downstream components of p53-mediated cell signal transduction pathways, miR-34b was discovered to serve a proapoptotic role in patients with DM complicated with HF $(138,139)$. It has been reported that p53 is a target of miR-30c and miE-181a (124). In addition, in another previous study, the downregulation of miR-30c and miR-181a in DCM was closely related to the activation of the p53 pathway in cardiomyocyte apoptosis (140).

During the course of DM, miRNAs have been discovered to be involved in numerous aspects of DCM pathogenesis, including apoptosis, fibrosis, hypertrophy, mitochondrial dysfunction and epigenetic modifications (141). Therefore, it is hypothesized that miRNAs may serve an important role in the pathophysiological processes of DCM. Further research into miRNAs has the potential to provide new perspectives into the prevention and treatment of DCM.

\section{Conclusions}

Oxidative stress is known to serve a significant role in DCM. Therefore, signaling via the NRF2/Keap1/ARE pathway may represent a novel drug target for the treatment of DCM. In addition, research into the relationship between miRNA and DCM has far-reaching clinical significance. Thus, the role of miRNA in the pathophysiology of DCM must be further clarified to be applied in clinical practice.

\section{Acknowledgements}

Not applicable.

\section{Funding}

The present study was supported by the National Natural Science Foundation of China (grant no. 00019509) and the Jiangxi Provincial Natural Science Foundation (grant no. 700207006).

\section{Availability of data and materials}

Not applicable.

\section{Authors' contributions}

XW and JL wrote the manuscript, reviewed the draft; and LH was responsible for analyzing the literature and reviewing drafts of the manuscript. All authors read and approved the final manuscript.

\section{Ethics approval and consent to participate}

Not applicable.

\section{Patient consent for publication}

Not applicable.

\section{Competing interests}

The authors declare that they have no competing interests.

\section{References}

1. Schrier RW, Abdallah JG, Weinberger HH and Abraham WT: Therapy of heart failure. Kidney Int 57: 1418-1425, 2000.

2. Rafeian-Kopaei M, Setorki M, Doudi M, Baradaran A and Nasri H: Atherosclerosis: Process, indicators, risk factors and new hopes. Int J Prev Med 5: 927-946, 2014.

3. Majerczyk M,Choręza P,Mizia-Stec K,Bożentowicz-Wikarek M, Brzozowska A, Arabzada H, Owczarek AJ, Szybalska A, Grodzicki T, Więcek A, et al: Plasma level of retinol-binding protein $4, \mathrm{~N}$-terminal proBNP and renal function in older patients hospitalized for heart failure. Cardiorenal Med 8: 237-248, 2018.

4. McGillicuddy FC, Moll HP, Farouk S, Damrauer SM, Ferran C and Reilly MP: Translational studies of A20 in atherosclerosis and cardiovascular disease. Adv Exp Med Biol 809: 83-101, 2014.

5. Lindblom R, Ververis K, Tortorella SM and Karagiannis TC: The early life origin theory in the development of cardiovascular disease and type 2 diabetes. Mol Biol Rep 42: 791-797, 2015.

6. Eltzschig HK and Eckle T: Ischemia and reperfusion-from mechanism to translation. Nat Med 17: 1391-1401, 2011.

7. Scolletta $S$ and Biagioli BE: Nergetic myocardial metabolism and oxidative stress: Let's make them our friends in the fight against heart failure. Biomed Pharmacother 64: 203-207, 2010.

8. Reczek CR and Chandel NS: ROS-dependent signal transduction. Curr Opin Cell Biol 33C: 8-13, 2014.

9. Ichihara S: The pathological roles of environmental and redox stresses in cardiovascular diseases. Environ Health Prev Med 18: 177-184, 2013.

10. Brahmanaidu P, Sathibabu U and Ganapathy S: Diabetic cardiomyopathy: Molecular mechanisms, detrimental effects of conventional treatment, and beneficial effects of natural therapy. Heart Fail Rev 24: 279-299, 2019.

11. Chakraborty S and Ain R: Nitric-oxide synthase trafficking inducer is a pleiotropic regulator of endothelial cell function and signaling. J Biol Chem 292: 6600-6620, 2017.

12. Balakumar P, Singh AP and Singh M: Rodent models of heart failure. J Pharmacol Toxicol Methods 56: 1-10, 2007.

13. Furfaro AL, Traverso N, Domenicotti C, Piras S, Moretta L, Marinari UM, Pronzato MA and Nitti M: The NRF2/HO-1 axis in cancer cell growth andchemoresistance. Oxid Med Cell Longev 2016: 1958174, 2016.

14. Suzuki T and Yamamoto M: Molecular basis of the Keap1-Nrf2 system. Free Radic Biol Med 88: 93-100, 2015.

15. Jay PY, Berul CI, Tanaka M, Ishii M, Kurachi Y and Izumo S: Cardiac conduction and arrhythmia: Insights from Nkx2.5 mutations in mouse and humans. Novartis Found Symp 250: 227-238, 2003.

16. Namani A, Li Y, Wang XJ and Tang X: Modulation of Nrf2 signaling pathway by nuclear receptors: Implications for cancer. Biochim Biophys Acta 1843: 1875-1885, 2014.

17. Yamamoto M, Kensler TW and Motohashi H: The KEAP1-NRF2 system: A thiol-based sensor-effector apparatus for maintaining redox homeostasis. Physiol Rev 98: 1169-1203, 2018.

18. Li W and Kong AN: Molecular mechanisms of Nrf2-mediated antioxidant response. Mol Carcinog 48: 91-104, 2009.

19. Nguyen T, Nioi P and Pickett CB: The Nrf2-antioxidant response element signaling pathway and its activation by oxidative stress. J Biol Chem 284: 13291-13295, 2009.

20. Corradi D, Callegari S, Maestri R, Benussi S and Alfieri O: Structural remodeling in atrial fibrillation. Nat Clin Pract Cardiovasc Med 5: 782-796, 2008. 
21. Saracino MR and Lampe JW: Phytochemical regulation of UDP-glucuronosyltransferases: Implications for cancer prevention. Nutr Cancer 59: 121-141, 2007.

22. Niture SK, Kaspar JW and Shen J: Nrf2 signaling and cell survival. Toxicol Appl Pharmacol 244: 37-42, 2010

23. Dhamodharan U, Ponjayanthi B, Sireesh D, Bhakkiyalakshmi E and Ramkumar KM: Association of single-nucleotide polymorphisms of the KEAP1 gene with the risk of various human diseases and its functional impact using in silico analysis. Pharmacol Res 137: 205-218, 2018.

24. Tian W, Rojo de la Vega M, Schmidlin CJ, Ooi A and Zhang DD: Kelch-like ECH-associated protein 1 (KEAP1) differentially regulates nuclear factor erythroid-2-related factors 1 and 2 (NRF1 and NRF2). J Biol Chem 293: 2029-2040, 2018

25. Mohan S and Gupta D: Crosstalk of toll-like receptors signaling and Nrf2 pathway for regulation of inflammation. Biomed Pharmacother 108: 1866-1878, 2018.

26. Niture SK, Khatri R and Jaiswal AK: Regulation of NRF2-an update. Free Radic Biol Med 66: 36-44, 2014.

27. Katoh Y, Iida K, Kang MI, Kobayashi A, Mizukami M, Tong KI, McMahon M, Hayes JD, Itoh K and Yamamoto M: Evolutionary conserved N-terminal domain of $\mathrm{Nrf2}$ is essential for the Keap1-mediated degradation of the protein by proteasome. Arch Biochem Biophys 433: 342-350, 2005.

28. Baird L, Llères D, Swift S and Dinkova-Kostova AT: Regulatory flexibility in the Nrf2-mediated stress response is conferred by conformational cycling of the Keap1-Nrf2 protein complex. Proc Natl Acad Sci USA 110: 15259-15264, 2013.

29. Hegedús K, Nagy P, Gáspári Z and Juhász G: The putative HORMA domain protein Atg101 dimerizes and is required for starvation-induced and selective autophagy in Drosophila. Biomed Res Int 2014: 470482, 2014.

30. Baird L and Dinkova-Kostova AT: The cytoprotective role of the Keap1-Nrf2 pathway. Arch Toxicol 85: 241-272, 2011.

31. Zhao Q, Liu Z and Huang B: PEDF improves cardiac function in rats subjected to myocardial ischemia/reperfusion injury by inhibiting ROS generation via PEDF-R. Int J Mol Med 41: 3243-3252, 2018

32. Sporn MB and Liby KT: NRF2 and cancer: The good, the bad and the importance of context. Nat Rev Cancer 12: 564-571, 2012.

33. Cameron BD, Sekhar KR, Ofori M and Freeman ML: The role of Nrf2 in the response to normal tissue radiation injury. Radiat Res 190: 99-106, 2018.

34. Xiang MJ, Namani A, Wu SJ and Wang XL: Nrf2: Bane or blessing in cancer? J Cancer Res Clin Oncol 140: 1251-1259, 2014.

35. Lau YS, Ling WC, Murugan D and Mustafa MR: Boldine ameliorates vascular oxidative stress and endothelial dysfunction: Therapeutic implication for hypertension and diabetes. J Cardiovasc Pharmacol 65: 522-531, 2015.

36. Gillet FX, Bournaud C, Antonino de Souza Júnior JD and Grossi-de-Sa MF: Plant-parasitic nematodes: Towards understanding molecular players in stress responses. Ann Bot 119: 775-789, 2017.

37. Lu MC, Ji JA, Jiang ZY and You QD: The Keap1-Nrf2-ARE pathway as a potential preventive and therapeutic target: An update. Med Res Rev 36: 924-963, 2016.

38. Cheng D, Wu R, Guo Y and Kong AN: Regulation of Keap1-Nrf2 signaling: The role of epigenetics. Curr Opin Toxicol 1: 134-138, 2016.

39. Chiou YS, Huang Q, Ho CT, Wang YJ and Pan MH: Directly interact with Keapl and LPS is involved in the anti-inflammatory mechanisms of (-)-epicatechin-3-gallate in LPS-induced macrophages and endotoxemia. Free Radic Biol Med 94: 1-16, 2016.

40. Itoh K, Ye P, Matsumiya T, Tanji K and Ozaki T: Emerging functional cross-talk between the Keap1-Nrf2 system and mitochondria. J Clin Biochem Nutr 56: 91-97, 2015.

41. Zenkov NK, Menshchikova EB and Tkachev VO Keap1/Nrf2/ARE redox-sensitive signaling system as a pharmacological target. Biochemistry (Mosc) 78: 19-36, 2013.

42. Tu J, Zhang X, Zhu Y, Dai Y, Li N, Yang F, Zhang Q, Brann DW and Wang R: Cell-permeable peptide targeting the Nrf2-Keap1 interaction: A potential novel therapy for global cerebral ischemia. Neurosci 35: 14727-14739, 2015.

43. Wakabayashi N, Slocum SL, Skoko JJ, Shin S and Kensler TW: When NRF2 talks, who's listening? Antioxid Redox Signal 13: $1649-1663,2010$
44. Thygesen K, Alpert JS, Jaffe AS, Simoons ML, Chaitman BR and White HD; Task Force for the Universal Definition of Myocardial Infarction: Third universal definition of myocardial infarction. Nat Rev Cardiol 9: 620-633, 2012.

45. Ostadal B, Drahota Z, Houstek J, Milerova M, Ostadalova I, Hlavackova M and Kolar F: Developmental and sex difference in cardiac tolerance to ischemia/reperfusion injury: The role of mitochondria ${ }^{1}$. Can J Physiol Pharmacol 97: 808-814, 2019.

46. Xue M, Momiji H, Rabbani N, Barker G, Bretschneider T, Shmygol A, Rand DA and Thornalley PJ: Frequency modulated translocational oscillations of Nrf2 mediate the antioxidant response element cytoprotective transcriptional response. Antioxid Redox Signal 23: 613-629, 2015.

47. Chai D, Zhang L, Xi S, Cheng Y, Jiang H and Hu R: Nrf2 activation induced by Sirt1 ameliorates acute lung injury after intestinal ischemia/reperfusion through NOX4-mediated gene regulation. Cell Physiol Biochem 46: 781-792, 2018.

48. Schwarz M, Lossow K, Kopp JF, Schwerdtle T and Kipp AP: Crosstalk of Nrf2 with the trace elements selenium, iron, zinc, and copper. Nutrients 11: 2112, 2019.

49. Mazzei L, Docherty NG and Manucha W: Mediators and mechanisms of heat shock protein 70 based cytoprotection in obstructive nephropathy. Cell Stress Chaperones 20: 893-906, 2015.

50. Mann GE, Bonacasa B, Ishii T and Siow RC: Targeting the redox sensitive Nrf2-Keap1 defense pathway in cardiovascular disease: Protection afforded by dietary isoflavones. Curr Opin Pharmacol 9: 139-145, 2009.

51. Xu B, Zhang J, Strom J, Lee S and Chen QM: Myocardial ischemic reperfusion induces de novo NRF2 protein translation. Biochim Biophys Acta 1842: 1638-1647, 2014.

52. Ashrafian H, Czibik G, Bellahcene M, Aksentijević D, Smith AC, Mitchell SJ, Dodd MS, Kirwan J, Byme JJ, Ludwiq C, et al: Fumarate is cardioprotective via activation of the NRF2 antioxidant pathway. Cell Metab 15: 361-371, 2012

53. Farías JG, Carrasco-Pozo C, Carrasco Loza R, Sepúlveda N, Álvarez P, Quezada M, Quiñones J, Molina V and Castillo RL: Polyunsaturated fatty acid induces cardioprotection against ischemia- reperfusion through the inhibition of NF-kappaB and induction of NRF2. Exp Biol Med 242: 1104-1114, 2017.

54. Bhogal RH, Weston CJ, Velduis S, G D Leuvenink H, Reynolds GM, Davies S, Nyguet-Thin L, Alfaifi M, Shepard EL, Boteon $\mathrm{Y}$, et al: The reactive oxygen species-mitophagy signaling pathway regulates liver endothelial cell survival during ischemia/reperfusion injury. Liver Transpl 24: 1437-1452, 2018.

55. Scherz-Shouval R and Elazar Z: ROS, mitochondria and the regulation of autophagy. Trends Cell Biol 17: 422-427, 2007.

56. Dinkova-Kostova AT and Abramov AY: The emerging role of NRF2 in mitochondrial function. Free Radic Biol Med 88: 179-188, 2015.

57. Ludtmann MH, Angelova PR, Zhang Y, Abramov AY and Dinkova-Kostova AT: NRF2 affects the efficiency of mitochondrial fatty acid oxidation. Biochem J 457: 415-424, 2014.

58. Glinka YY and Youdim MB: Inhibition of mitochondrial complexes I and IV by 6-hydroxydopamine. Eur J Pharmacol 292: 329-332, 1995.

59. Baechler BL, Bloemberg D and Quadrilatero J: Mitophagy regulates mitochondrial network signaling, oxidative stress, and apoptosis during myoblast differentiation. Autophagy 15: 1606-1619, 2019

60. Qiu M,Zhang S, Ke L, Tang H, Zeng X and Liu J: JS-K enhances chemosensitivity of prostate cancer cells to Taxol via reactive oxygen species activation. Oncol Lett 17: 757-764, 2019.

61. Zhang Y, Sano M, Shinmura K, Tamaki K, Katsumata Y, Matsuhashi T, Morizane S, Ito H, Hishiki T, Endo J, et al: 4-hydroxy-2-nonenal protects against cardiac ischemiareperfusion injury via the NRF2-dependent pathway. J Mol Cell Cardiol 49: 576-586, 2010.

62. Anedda A, López-Bernardo E, Acosta-Iborra B, Saadeh Suleiman M, Landázuri MO and Cadenas S: The transcription factor NRF2 promotes survival by enhancing the expression of uncoupling protein 3 under conditions of oxidative stress. Free Radic Biol Med 61C: 395-407, 2013.

63. Curfman G: Stem cell therapy for heart failure: An unfulfilled promise? JAMA 321: 1186-1187, 2019.

64. Chen YL, Fan J, Cao L, Han TL, Zeng M, Xu Y, Ling Z and Yin Y: Unique mechanistic insights into the beneficial effects of angiotensin-(1-7) on the prevention of cardiac fibrosis: A metabolomic analysis of primary cardiac fibroblasts. Exp Cell Res 378: 158-170, 2019. 
65. Ambrosi N, Guerrieri D, Caro F, Sanchez F, Haeublein G, Casadei D, Incardona $C$ and Chuluyan E: Alpha lipoic acid: A therapeutic strategy that tend to limit the action of free radicals in transplantation. Int J Mol Sci 19: 102, 2018.

66. Kageyama S, Saito T, Obata M, Koide RH, Ichimura Y and Komatsu M: Negative regulation of the Keap1-Nrf2 pathway by a p62/Sqstm1 splicing variant. Mol Cell Biol 38: e00642-17, 2018.

67. Erpicum P, Rowart P, Defraigne JO, Krzesinski JM and Jouret F: What we need to know about lipid-associated injury in case of renal ischemia-reperfusion. Am J Physiol Renal Physiol 315 : F1714-F1719, 2018.

68. Lee LY, Harberg C, Matkowskyj KA, Cook S, Roenneburg D, Werner S, Johnson J and Foley DP: Overactivation of the nuclear factor (erythroid-derived 2)-like 2-antioxidant response element pathway in hepatocytes decreases hepatic ischemia/reperfusion injury in mice. Liver Transpl 22: 91-102, 2015.

69. Kaplinsky E: DAPA-HF trial: Dapagliflozin evolves from a glucose-lowering agent to a therapy for heart failure. Drugs Context 9: 2019-11-3, 2020.

70. Virani SA, Sharma V, McCann M, Koehler J, Tsang B and Zieroth S: Prospective evaluation of integrated device diagnostics for heart failure management: Results of the TRIAGE-HF study. ESC Heart Fail 5: 809-817, 2018.

71. McMurray JJ, Adamopoulos S, Anker SD, Auricchio A, Böhm M, Dickstein K, Falk V, Filippatos G, Fonseca C, Gomez-Sanchez MA, et al: ESC guidelines for the diagnosis and treatment of acute and chronic heart failure 2012: The task force for the diagnosis and treatment of acute and chronic heart failure 2012 of the European Society of Cardiology. Developed in collaboration with the Heart Failure Association (HFA) of the ESC. Eur Heart J 33: 1787-1847, 2012.

72. Zhu X, Oseghale AR, Nicole LH, Li B and Pace BS: Mechanisms of NRF2 activation to mediate fetal hemoglobin induction and protection against oxidative stress in sickle cell disease. Exp Biol Med (Maywood) 244: 171-182, 2019.

73. Rajasekaran NS, Varadharaj S, Khanderao GD, Davidson CJ, Kannan S, Firpo MA, Zweier JL and Benjamin IJ: Sustained activation of nuclear erythroid 2-related factor 2/antioxidant response element signaling promotes reductive stress in the human mutant protein aggregation cardiomyopathy in mice. Antioxid Redox Signal 14: 957-971, 2011

74. Ichikawa T, Li J, Meyer CJ, Janicki JS, Hannink M and Cui T: Dihydro-CDDO-trifluoroethyl amide (dh404), a novel Nrf2 activator, suppresses oxidative stress in cardiomyocytes. PLoS One 4: e8391, 2009.

75. Li J, Zhang C, Xing Y, Janicki JS, Yamamoto M, Wang XL, Tang DQ and Cui T: Up-regulation of p27(kip1) contributes to Nrf2-mediated protection against angiotensin II-induced cardiac hypertrophy. Cardiovasc Res 90: 315-324, 2011.

76. Burchfield JS, Xie M and Hill JA: Pathological ventricular remodeling: Mechanisms: Part 1 of 2. Circulation 128: 388-400, 2013.

77. Takimoto E and Kass DA: Role of oxidative stress in cardiac hypertrophy and remodeling. Hypertension 49: 241-248, 2007.

78. Guan Y, Zhou L, Zhang Y, Tian H, Li A and Han X: Effects of PP2A/Nrf2 on experimental diabetes mellitus-related cardiomyopathy by regulation of autophagy and apoptosis through ROS dependent pathway. Cell Signal 62: 109339, 2019.

79. Murdoch CE, Zhang M, Cave AC and Shah AM: NADPH oxidase-dependent redox signalling in cardiac hypertrophy, remodelling and failure. Cardiovasc Res 71: 208-215, 2006.

80. Shuai W, Kong B, Fu H, Shen C, Jiang X and Huang H: MD1 Deficiency promotes inflammatory atrial remodelling induced by high-fat diets. Can J Cardiol 35: 208-216, 2019.

81. Yu C, Lin H, Yang H, Kong SL, Zhang Q and Lee SW Progression of systolic abnormalities in patients with 'isolated' diastolic heart failure and diastolic dysfunction. Circulation 105 $1195-1201,2002$

82. Cai L, Li W, Wang G, Guo L, Jiang Y and Kang YJ Hyperglycemia-induced apoptosis in mouse myocardium: Mitochondrial cytochrome C-mediated caspase-3 activation pathway. Diabetes 51: 1938-1948, 2002.

83. Fassett J, Xu X, Kwak D, Zhu G, Fassett EK, Zhang P, Wang H, Maver B, Bache RJ and Chen Y: Adenosine kinase attenuates cardiomyocyte microtubule stabilization and protects against pressure overload-induced hypertrophy and LV dysfunction. J Mol Cell Cardiol 130: 49-58, 2019.

84. Hafstad AD, Nabeebaccus AA and Shah AM: Novel aspects of ROS signalling in heart failure. Basic Res Cardiol 108: 359, 2013.
85. Gupta S, Das B and Sen S: Cardiac hypertrophy: Mechanisms and therapeutic opportunities. Antioxid Redox Signal 9: 623-652, 2007.

86. Sabri A, Hughie HH and Lucchesi PA: Regulation of hypertrophic and apoptotic signaling pathways by reactive oxygen species in cardiac myocytes. Antioxid Redox Signal 5: 731-740, 2003.

87. Zhang X, Xiao Z, Yao J, Zhao G, Fa X and Niu J: Participation of protein kinase $\mathrm{C}$ in the activation of $\mathrm{Nrf} 2$ signaling by ischemic preconditioning in the isolated rabbit heart. Mol Cell Biochem 372: 169-179, 2013 .

88. Zhao WY, Zhao TQ, Chen YJ, Ahokas RA and Sun Y: Oxidative stress mediates cardiac fbrosis by enhancing transforming growth factor-betal in hypertensive rats. Mol Cell Biochem 317: 43-50, 2008

89. Yang T, Sun Y, Mao L, Zhang M, Li Q, Zhang L, Shi Y, Leak RK, Chen $\mathrm{J}$ and Zhang F: Brain ischemic preconditioning protects against ischemic injury and preserves the blood-brain barrier via oxidative signaling and Nrf2 activation. Redox Biol 17: 323-337, 2018

90. James PA, Oparil S, Carter BL, Cushman WC, DennisonHimmelfarb C, Handler J, Lackland DT, LeFevre ML, MacKenzie TD, Oqedeqbe O, et al: Evidence-based guideline for the management of high blood pressure in adults: Report from the panel members appointed to the Eighth Joint National Committee. JAMA 311: 507-520, 2014.

91. Hu CM, Chen YH, Chiang MT and Chau LY: Heme oxygenase- 1 inhibits angiotensin II-induced cardiac hypertrophy in vitro and in vivo. Circulation 110: 309-316, 2004.

92. Mancusi C, Canciello G, Izzo R, Damiano S, Grimaldi MG, Luca N, Simone G, Trimarco B and Losi MA: Left atrial dilatation: A target organ damage in young to middle-age hypertensive patients. The Campania Salute Network. Int J Cardiol 265: 229-233, 2018.

93. WangZH,LiuJL, WuL, YuZand YangHT:Concentration-dependent wrestling between detrimental and protective effects of $\mathrm{H}_{2} \mathrm{O}_{2}$ during myocardial ischemia/reperfusion. Cell Death Dis 5: e1297, 2014.

94. Yao SY, Liu J, Li Y, Wang M, Wang C and Xue H: Association between plasma microRNA-29a and left ventricular hypertrophy in patients with hypertension. Zhonghua Xin Xue Guan Bing Za Zhi 47: 215-220, 2019 (In Chinese)

95. Mitra A, Basak T, Datta K, Naskar S, Sengupta S and Sarkar S: Role of a-crystallin B as a regulatory switch in modulating cardiomyocyte apoptosis by mitochondria or endoplasmic reticulum during cardiac hypertrophy and myocardial infarction. Cell Death Dis 4: e582, 2013.

96. Delmar M and Makita N: Cardiac connexins, mutations and arrhythmias. Curr Opin Cardiol 27: 236-241, 2012.

97. Stout JM, Gousset MU, Drummond HA, Gray W III, Pruett BE and Stec DE: Sex-specific effects of heme oxygenase-2 deficiency on renovascular hypertension. J Am Soc Hypertens 7: 328-335, 2013

98. Tian C, Gao L, Zimmerman MC and Zucker IH: Myocardial infarction-induced microRNA-enriched exosomes contribute to cardiac Nrf2 dysregulation in chronic heart failure. Am J Physiol Heart Circ Physiol 314: H928-H939, 2018

99. Tham YK, Bernardo BC, Ooi JY, Weeks KL and McMullen JR Pathophysiology of cardiac hypertrophy and heart failure: Signaling pathways and novel therapeutic targets. Arch Toxicol 89: 1401-1438, 2015.

100. Yang KC and Dudley JSC: Oxidative stress and atrial fibrillation: Finding a missing piece to the puzzle. Circulation 128: 1724-1726, 2013.

101. Barry SP, Davidson SM and Townsend PA: Molecular regulation of cardiac hypertrophy. Int J Biochem Cell Biol 40: 2023-2039, 2008.

102. Maulik SK and Kumar S: Oxidative stress and cardiac hypertrophy: A review. Toxicol Mech Methods 22: 359-366, 2012.

103. Baruteau AE, Probst V and Abriel H: Inherited progressive cardiac conduction disorders. Curr Opin Cardiol 30: 33-39, 2015.

104. Chang YJ, Hsiao HJ, Hsia SH, Lin JJ, Hwang MS, Chung HT, Chen CL, Huang YC and Tsai MH: Analysis of clinical parameters and echocardiography as predictors of fatal pediatric myocarditis. PLoS One 14: e0214087, 2019.

105. Zhao YS, An JR, Yang S, Guan P, Yu FY, Li W, Li JR, Guo Y, Sun ZM and Ji ES: Hydrogen and oxygen mixture to improve cardiac dysfunction and myocardial pathological changes induced by intermittent hypoxia in rats. Oxid Med Cell Longev 2019: 7415212, 2019. 
106. Nakamura M and Sadoshima J: Mechanisms of physiological and pathological cardiac hypertrophy. Nat Rev Cardiol 15: 387-407, 2018.

107. Li J, Ichikawa T, Villacorta L, Janicki JS, Brower GL, Yamamoto $\mathrm{M}$ and Cui T: Nrf2 protects against maladaptive cardiac responses to hemodynamic stress. Arterioscler Thromb Vasc Biol 29: 1843-1850, 2009.

108. Nakamura M and Sadoshima J: Cardiomyopathy in obesity, insulin resistance or diabetes. J Physiol 598: 2977-2993, 2020.

109. Felker GM, Thompson RE, Hare JM, Hruban RH, Clemetson DE, Howard DL, Baughman K and Kasper EK: Underlying causes and long-term survival in patients with initially unexplained cardiomyopathy. N Engl J Med 342: 1077-1184, 2000

110. Quinaglia T, Oliveira DC, Matos-Souza JR and Sposito AC: Diabetic cardiomyopathy: Factual or factoid? Rev Assoc Med Bras (1992) 65: 61-69, 2019.

111. Cooper LT Jr: Myocarditis. N Engl J Med 360: 1526-1538, 2009.

112. Althunibat OY, Al Hroob AM, Abukhalil MH, Germoush MO, Bin-Jumah M and Mahmoud AM: Fisetin ameliorates oxidative stress, inflammation and apoptosis in diabetic cardiomyopathy. Life Sci 221: 83-92, 2019.

113. Zhao X, Cai A, Peng Z, Liang W, Xi H, Li P, Chen G, Yu J and Chen L: JS-K induces reactive oxygen species-dependent anti-cancer effects by targeting mitochondria respiratory chain complexes in gastric cancer. J Cell Mol Med 23: 2489-2504, 2019

114. Ansley DM and Wang B: Oxidative stress and myocardial injury in the diabetic heart. J Pathol 229: 232-241, 2013.

115. Hernández M, Wicz S and Corral RS: Cardioprotective actions of curcumin on the pathogenic NFAT/COX-2/prostaglandin E2 pathway induced during Trypanosoma cruzi infection. Phytomedicine 23: 1392-1400, 2016.

116. Ndisang JF, Lane N, Syed N and Jadhav A: Up-regulating the heme oxygenase system with hemin improves insulin sensitivity and glucose metabolism in adult spontaneously hypertensive rats. Endocrinology 151: 549-560, 2010.

117. Jiménez-Osorio AS, García-Niño WR, González-Reyes S, Álvarez-Mejía AE, Guerra-León S, Salazar-Segovia J, Falcón I, Montes de Oca-Solano H, Madero M and Pedraza-Chaverri J: The effect of dietary supplementation with curcumin on redox status and Nrf2 activation in patients with nondiabetic or diabetic proteinuric chronic kidney disease: A pilot study. J Ren Nutr 26: 237-244, 2016.

118. Soundararajan P and Kim JS: Anti-carcinogenic glucosinolates in cruciferous vegetables and their antagonistic effects on prevention of cancers. Molecules 23: 2983, 2018.

119. Wang J, Wang S, Wang W, Chen J, Zhang Z, Zheng Q, Liu Q and Cai L: Protection against diabetic cardiomyopathy is achieved using a combination of sulforaphane and zinc in type 1 diabetic OVE26 mice. J Cell Mol Med 23: 6319-6330, 2019.

120.Zhang Z, Wang S, Zhou S, Yan X, Wang Y, Chen J, Mellen N, Kong M, Gu J, Tan Y, et al: Sulforaphane prevents the development of cardiomyopathy in type 2 diabetic mice probably by reversing oxidative stress-induced inhibition of LKB1/AMPK pathway. J Mol Cell Cardiol 77: 42-52, 2014.

121. Bai Y, Wang X, Zhao S, Ma C, Cui J and Zheng Y: Sulforaphane protects against cardiovascular disease via Nrf2 activation. Oxid Med Cell Longev 2015: 407580, 2015.

122. Delucchi F, Berni R, Frati C, Cavalli S, Graiani G, Sala R, Chaponnier C, Gabbiani G, Calani L, Rio DD, et al: Resveratrol treatment reduces cardiac progenitor cell dysfunction and prevents morpho-functional ventricular remodeling in type-1 diabetic rats. PLoS One 7: e39836, 2012.

123. Sun X, Shan A, Wei Z and Xu B: Intravenous mesenchymal stem cell-derived exosomes ameliorate myocardial inflammation in the dilated cardiomyopathy. Biochem Biophys Res Commun 503: 2611-2618, 2018

124. Ge ZD, Lian Q, Mao X and Xia Z: Current status and challenges of NRF2 as a potential therapeutic target for diabetic cardiomyopathy. Int Heart J 60: 512-520, 2019.
125.El-Agamy DS, El-Harbi KM, Khoshhal S, Ahmed N, Elkablawy MA, Shaaban AA and Abo-Haded HM: Pristimerin protects against doxorubicin-induced cardiotoxicity and fibrosis through modulation of Nrf2 and MAPK/NF- $\mathrm{KB}$ signaling pathways. Cancer Manag Res 11: 47-61, 2018.

126. Zhou S, Jin J, Bai T, Sachleben LR Jr, Cai L and Zheng Y: Potential drugs which activate nuclear factor E2-related factor 2 signaling to prevent diabetic cardiovascular complications: A focus on fumaric acid esters. Life Sci 134: 56-62, 2015.

127. Saidu NE, Noé G, Cerles O, Cabel L, Kavian-Tessler N, Chouzenoux S, Bahuaud M, Chéreau C, Nicco C, Leroy K, et al: Dimethyl fumarate controls the NRF2/DJ-1 axis in cancer cells: Therapeutic applications. Mol Cancer Ther 16: 529-539, 2017.

128. Brennan MS, Patel H, Allaire N, Thai A, Cullen P, Rvan S, Lukashev M, Bista P, Huang R, Rhodes KJ and Scannevin RH: Pharmacodynamics of dimethyl fumarate are tissue specific and involve Nrf2-dependent and -independent mechanisms. Antioxid Redox Signal 24: 1058-1071, 2016.

129. Bomprezzi R: Dimethyl fumarate in the treatment of relapsing-remitting multiple sclerosis: An overview. Ther Adv Neurol Disord 8: 20-30, 2015.

130. Ganeshpurkar A and Saluja AK: The pharmacological potential of rutin. Saudi Pharm J 25: 149-164, 2017.

131. Moore PK, Griffiths RJ and Lofts FJ: The effect of some flavone drugs on the conversion of prostacyclin to 6-oxoprostaglandin E1. Biochem Pharmacol 32: 2813-2817, 1983.

132. Gao HC, Zhu K, Gao HM, Miao CS, Zhang LN, Liu W and $\mathrm{Xin} \mathrm{H}$ : Role of tissue transglutaminase in the pathogenesis of diabetic cardiomyopathy and the intervention effect of rutin. Exp Ther Med 9: 1103-1108, 2015.

133. Sayed AS, Xia K, Salma U, Yang T and Peng J: Diagnosis, prognosis and therapeutic role of circulating miRNAs in cardiovascular diseases. Heart Lung Circ 23: 503-510, 2014

134. Chen S, Puthanveetil P, Feng B, Matkovich SJ, Dorn GW II and Chakrabarti S: Cardiac miR-133a overexpression prevents early cardiac fibrosis in diabetes. J Cell Mol Med 18: 415-421, 2014.

135. Chen K, Ma Y, Wu S, Zhuang Y, Liu X, Lv L and Zhang G: Construction and analysis of a lncRNA-miRNA-mRNA network based on competitive endogenous RNA reveals functional lncRNAs in diabetic cardiomyopathy. Mol Med Rep 20: 1393-1403, 2019.

136. Lin $\mathrm{M}$ and Mao ZJ: IncRNA-mRNA competing endogenous RNA network in IR-hepG2 cells ameliorated by APBBR decreasing ROS levels: A systematic analysis. PeerJ 8: e8604, 2020.

137. Dludla PV, Nkambule BB, Dias SC and Johnson R: Cardioprotective potential of $\mathrm{N}$-acetyl cysteine against hyperglycaemia-induced oxidative damage: A protocol for a systematic review. Syst Rev 6: 96, 2017.

138. Mansueto G, Benincasa G, Della Mura N, Nicoletti GF and Napoli C: Epigenetic-sensitive liquid biomarkers and personalised therapy in advanced heart failure: A focus on cell-free DNA and microRNAs. J Clin Pathol 73: 535-543, 2020.

139. Zhang X, Dong S, Jia Q, Zhang A, Li Y, Zhu Y, Lv S and Zhang J: The microRNA in ventricular remodeling: The miR-30 family. Biosci Rep 39: BSR20190788, 2019.

140. Raut SK, Singh GB, Rastogi B, Saikia UN, Mittal A, Dogra N, Singh S, Prasad R and Khullar M: miR-30c and miR-181a synergistically modulate p53-p21 pathway in diabetes induced cardiac hypertrophy. Mol Cell Biochem 417: 191-203, 2016.

141. Li M, Chen X, Chen L, Chen K, Zhou J and Song J: MiR-1-3p that correlates with left ventricular function of HCM can serve as a potential target and differentiate HCM from DCM. J Transl Med 16: 161, 2018.

This work is licensed under a Creative Commons Attribution-NonCommercial-NoDerivatives 4.0 International (CC BY-NC-ND 4.0) License. 